\title{
Casca de ovo como fonte de cálcio para humanos: composição mineral e análise microbiológica
}

\author{
Eggshell as calcium source for humans: mineral composition and microbiological analysis
}

\author{
Bruna Gressler Milbradt ${ }^{I}$ Aline Lima Hermes MüllerII Jéssica Soares da Silva ${ }^{\text {III }}$ \\ Julianna Rodrigues Lunardi ${ }^{I I I}$ Liana Inês Guidolin Milani ${ }^{\mathrm{IV}}$ Érico Marlon de Moraes Flores ${ }^{\mathrm{V}}$ \\ Maria da Graça Kolisnki Callegaro ${ }^{\mathrm{III}}$ Tatiana Emanuelli ${ }^{\mathrm{II}}{ }^{*}$
}

\section{RESUMO}

Este estudo teve como objetivo avaliar a composição mineral de diferentes tipos de cascas de ovo, bem como a segurança microbiológica de amostras submetidas a diferentes métodos de higienização. Para a obtenção do pó de casca de ovo, as cascas foram lavadas, higienizadas, secas em estufa e trituradas em moinho. Cascas de ovo de granja (criação confinada), de coloração branca e vermelha, e cascas de ovo coloniais (caipira), provenientes da região central do Rio Grande do Sul, foram comparadas quanto a sua composição mineral. O Ca, mineral predominante na casca de ovo, se manteve em concentrações semelhantes nas diferentes amostras (cerca de $365 \mathrm{mg}^{-1}$ ). As cascas de ovo de granja apresentaram maior concentração de $\mathrm{Mg}$ e menor concentração de $\mathrm{Sr}$ que as cascas de ovo coloniais. Não foram encontradas quantidades significativas de $\mathrm{Fe}, \mathrm{Cr}, \mathrm{Mn}$, $\mathrm{Mo}, \mathrm{Ni}$, Se, Al, Cd e Pb nas amostras analisadas. Adicionalmente, tanto amostras higienizadas com imersão em hipoclorito $e$ posterior fervura em água, quanto amostras nas quais a imersão em hipoclorito foi suprimida, não apresentaram contaminação por coliformes, estafilococos ou salmonela. Os resultados indicam que a casca de ovo pode ser utilizada na nutrição humana, já que é rica em Ca, não apresenta contaminação por metais tóxicos e, se processada de forma adequada, apresenta boa qualidade higiênico-sanitária.

Palavras-chave: casca de ovo, cálcio, minerais, metais tóxicos, qualidade higiênico-sanitária.

\section{ABSTRACT}

This study aimed to evaluate the mineral composition of different kinds of eggshell, as well as the microbiological safety of samples submitted to different sanitization procedures. To obtain the eggshell powder, the shells were washed, sanitized, oven dried and grinded in a mill. White-and brown-colored eggshells from confined laying hens and eggshells from free-ranged laying hens from the central region of Rio Grande do Sul were compared for their mineral composition. $\mathrm{Ca}$, the predominant mineral in eggshells, remained at similar concentrations in the different samples (approximately $365 \mathrm{mg} \mathrm{g}^{-1}$ ). Eggshells from confined laying hens had higher $\mathrm{Mg}$ concentration and lower $\mathrm{Sr}$ concentration than the shells from free-ranged laying hens. No significant amounts of $\mathrm{Fe}, \mathrm{Cr}, \mathrm{Mn}$, $\mathrm{Mo}, \mathrm{Ni}$, Se, Al, Cd or Pb were found in the samples. Additionally, both samples that were sanitized by immersion in hypochlorite and subsequently boiled in water as well as samples in which hypochlorite immersion was suppressed did not show coliform, staphylococcus or salmonella contamination. The results indicate that eggshell can be used in human nutrition since it is rich $\mathrm{Ca}$ source, shows no contamination by toxic metals and has good sanitary quality when properly processed.

Key words: eggshells, calcium, minerals, toxic metals, sanitary quality.

\section{INTRODUÇÃO}

O cálcio (Ca) é o mineral mais abundante no corpo humano, sendo responsável por cerca de 1 a $2 \%$ do peso corporal (SILVA \& COZZOLINO, 2007). Além da sua função estrutural, uma vez que $99 \%$ de sua concentração

IPrograma de Pós-graduação em Ciência e Tecnologia dos Alimentos, Universidade Federal de Santa Maria (UFSM), Santa Maria, RS, Brasil.

"Programa de Pós-graduação em Química, UFSM, Santa Maria, RS, Brasil.

IIINúcleo Integrado de Desenvolvimento em Análises Laboratoriais (NIDAL), Departamento de Tecnologia e Ciência dos Alimentos (DTCA), UFSM, Camobi, 97105-900, Santa Maria, RS, Brasil. E-mail: tatiemanuelli@gmail.com. *Autor para correspondência.

IV Laboratório de Microbiologia, DTCA, UFSM, Santa Maria, RS, Brasil.

vDepartamento de Química, UFSM, Santa Maria, RS, Brasil. 
encontra-se nos ossos e dentes, este elemento é essencial na contração muscular, propagação do impulso nervoso, coagulação sanguínea, entre outras funções (AWUMEY \& BUKOSKI, 2006). A ingestão adequada de Ca é importante para atingir e preservar a massa óssea ideal, além de estar associada à menor prevalência de doenças crônicas (SCHULZE, 2013), entretanto, ela está frequentemente abaixo do recomendado. No Brasil, aproximadamente $90 \%$ da população idosa não alcança o requerimento médio estimado deste mineral através da alimentação (FISBERG et al., 2013).

A casca de ovo de galinha, que contém cerca de $40 \%$ de $\mathrm{Ca}$ na forma de carbonato de cálcio $\left(\mathrm{CaCO}_{3}\right)$, poderia ser uma opção de baixo custo e fácil obtenção para atender as necessidades nutricionais da população (SCHAAFSMA et al., 2000; ROVENSKÝ et al., 2003). Anualmente, cerca de 6 milhões de toneladas de cascas de ovo são geradas nas residências e em empresas alimentícias de todo o mundo (OLIVEIRA et al., 2013). A maior parte dessa produção é destinada atualmente à agricultura para correção do $\mathrm{pH}$ do solo (KING'ORI, 2011). A valorização deste subproduto pode reduzir a extração de reservas naturais não-renováveis de $\mathrm{CaCO}_{3}$, como rocha calcária (OLIVEIRA et al., 2013). Além disso, é importante ressaltar que o $\mathrm{Ca}$ da casca de ovo possui elevada biodisponibilidade, demonstrada tanto em animais experimentais quanto em humanos (SCHAAFSMA et al., 2002; NAVES, 2003; MAEHIRA et al., 2009; BRUN et al., 2013).

Além do $\mathrm{Ca}$, mineral mais abundante na casca de ovo, outros minerais podem ser encontrados em menores concentrações, como magnésio $(\mathrm{Mg})$, estrôncio $(\mathrm{Sr})$, ferro $(\mathrm{Fe})$, selênio (Se), entre outros (ROVENSKÝ et al., 2003). No entanto, somente alguns estudos avaliaram a composição mineral deste produto, utilizando número expressivo de amostras (SCHAAFSMA et al., 2000; KÜÇÜKYILMAZ et al., 2012; BRUN et al., 2013).

O sistema de criação das poedeiras é um possível fator de influência sobre a composição mineral de cascas de ovo (SCHAAFSMA et al., 2000; KÜÇÜKYILMAZ et al., 2012). Atualmente, a maioria dos ovos disponíveis para comercialização provém de galinhas poedeiras criadas em granjas no sistema intensivo (confinadas em gaiolas), sendo que a variação mais perceptível destes encontra-se na coloração das cascas (classificados em brancos e vermelhos).
Por outro lado, os ovos designados coloniais ou caipiras estão sendo bastante valorizados e provêm de galinhas poedeiras criadas em liberdade (sistema extensivo ou semiextensivo). Ao contrário dos ovos de granja, os ovos coloniais não apresentam uma categorização por cor, em função da variabilidade genética das poedeiras. No Brasil, existem estudos avaliando o teor de $\mathrm{Ca}$ e outros minerais em amostra de casca do ovo (VILAR et al., 2010; SANTOS et al., 2012), mas não há comparação entre amostras de diferentes sistemas de criação, coloração e origens. Por sua vez, a relação entre a coloração da casca e sua composição mineral ainda não foi estudada.

A segurança de suplementos naturais de $\mathrm{Ca}$ tem sido questionada devido à possibilidade de contaminação por elementos tóxicos como chumbo $(\mathrm{Pb})$, cádmio $(\mathrm{Cd})$ e mercúrio $(\mathrm{Hg})$ (KIM, 2004; MATTOS et al., 2006). No Brasil, apenas VILAR et al. (2010) e SANTOS et al. (2012) avaliaram níveis de metais tóxicos em amostra de cascas de ovo. Outro aspecto que deve ser considerado para o uso de casca de ovo por humanos é sua segurança microbiológica, por se tratar de um produto suscetível à contaminação por bactérias, sobretudo do gênero Salmonella (NAVES et al., 2007).

O presente estudo teve como objetivos avaliar a influência do sistema de criação das galinhas poedeiras e coloração das cascas na composição de minerais essenciais e tóxicos e a influência da higienização com hipoclorito de sódio sobre a segurança microbiológica de pó de cascas de ovo, provenientes da região central do Rio Grande do Sul.

\section{MATERIAL E MÉTODOS}

Para a análise de minerais, foram adquiridas, entre julho e novembro de 2011, em mercados e feiras da região central do Rio Grande do Sul, 28 amostras, sendo cada unidade experimental composta de 24 unidades de ovos. Os ovos foram procedentes de galinhas poedeiras mantidas confinadas em gaiolas (ovos de granja), nas colorações de casca branca $(\mathrm{N}=11)$ e vermelha $(\mathrm{N}=9)$ e de galinhas poedeiras criadas em liberdade, em pequenas propriedades rurais (ovos coloniais) ( $\mathrm{N}=8$ ). Os ovos coloniais não foram separados por coloração, já que há grande variação entre colorações e tonalidades, diferindo mesmo entre as unidades que formavam uma amostra. As etapas envolvidas na obtenção 
dos pós de cascas de ovo estão apresentadas na figura 1.

Amostras de pó de casca de ovo (em triplicata) foram digeridas com ácido nítrico (Merck, Alemanha) em bloco digestor e diluídas com água milli-Q. $\mathrm{Ca}, \mathrm{Mg}$ e $\mathrm{Sr}$ foram analisados por espectrometria de absorção atômica (AAS) de alta resolução (ContrAA 700 Analytikjena). Cd, Pb, Cromo (Cr), Manganês (Mn), Molibdênio (Mo), Níquel (Ni), e Se foram determinados utilizando espectrômetro de massas com plasma indutivamente acoplado (ICP-MS) (Perkin-Elmer-SCIEX, Elan DRC II). Fe e Alumínio (Al) foram determinados utilizando espectrômetro de emissão óptica com plasma indutivamente acoplado (ICP-OES) (Spectro Ciros CCD).

A qualidade microbiológica das cascas de ovo foi testada em três das amostras utilizadas para a análise de minerais após higienização por imersão em hipoclorito de sódio $1 \%(0,48 \%$ de cloro livre) durante $5 \mathrm{~min}$., seguido de fervura em água por 10min. (tratamento 1; higienização conforme NAVES et al., 2007). Utilizou-se uma proporção de peso de casca para líquido de aproximadamente 1:10. Para avaliar se a imersão em hipoclorito poderia ser dispensável na higienização, três amostras adicionais foram preparadas excluindo esta etapa (tratamento 2). A avaliação microbiológica consistiu na contagem de Staphylococcus coagulase positiva, determinação de coliformes totais e termotolerantes e pesquisa de Salmonella sp., conforme os métodos oficiais (BRASIL, 2003a). A interpretação dos resultados foi feita de acordo com os critérios microbiológicos para ovos estabelecidos na legislação brasileira (BRASIL, 2001).

Os resultados de composição mineral das cascas foram submetidos à análise de variância de uma via (ANOVA) e as diferenças entre os grupos foram avaliadas usando teste de Duncan, considerando o nível de 5\% de significância. O software empregado foi Statistica 6.0 para Windows.

\section{RESULTADOS E DISCUSSÃO}

$\mathrm{Na}$ tabela 1 é apresentado o conteúdo de minerais essenciais e não essenciais de cascas de ovo de granja, brancas e vermelhas, e coloniais. Neste estudo, não foram observadas diferenças na composição mineral entre os ovos de granja brancos e vermelhos, possivelmente em função da maior diferença entre essas cascas ser na sua composição orgânica (MINE, 2008). Por outro lado, ocorreram

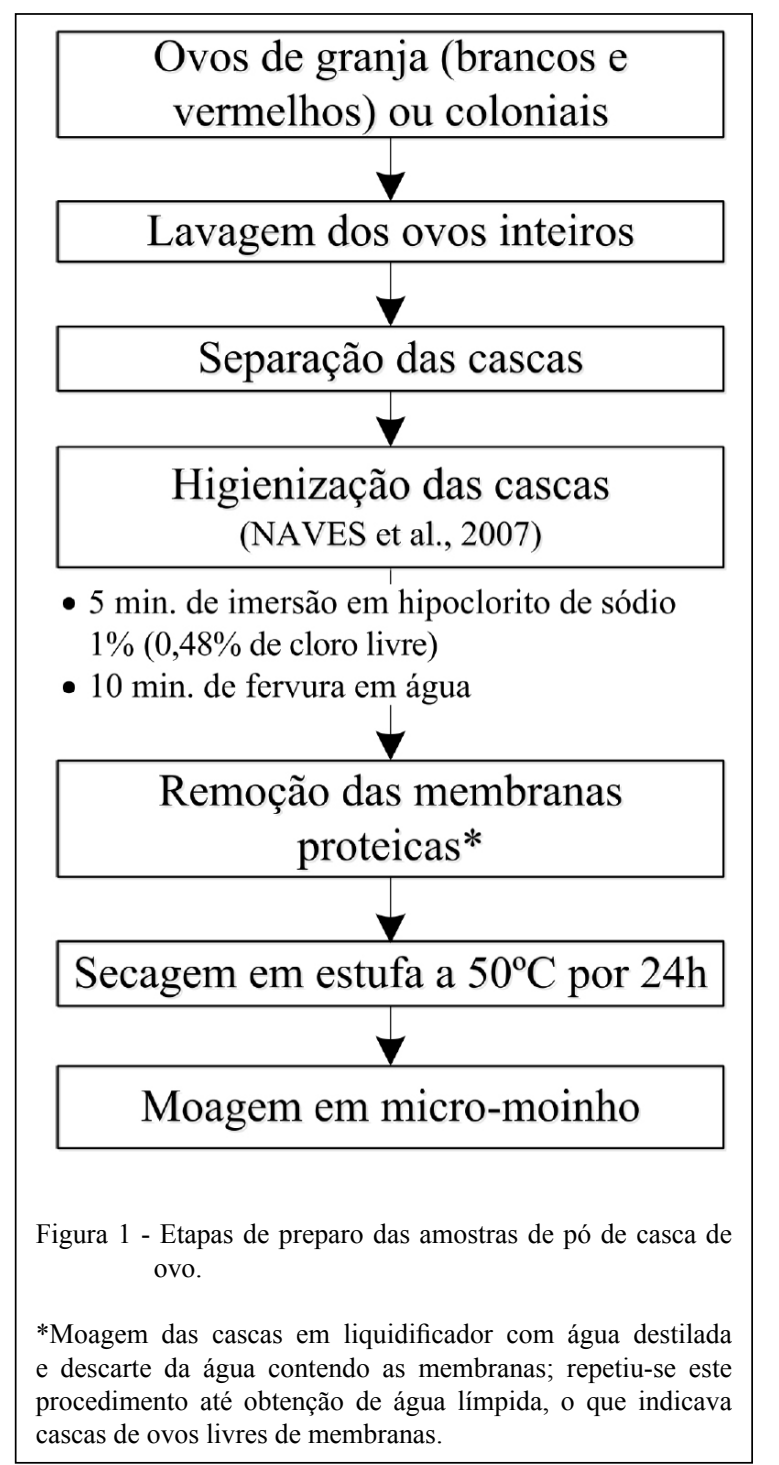

pequenas diferenças de composição mineral entre cascas de ovo coloniais e de granja que serão discutidas a seguir.

A concentração de $\mathrm{Ca}$ atingiu $37 \%$ do pó de casca de ovo em média em todas as amostras analisadas $\left(365,7 \mathrm{mg} \mathrm{g}^{-1}\right.$; Tabela 1). Este teor foi semelhante às concentrações encontradas por SCHAAFSMA et al. (2000), em média $390 \mathrm{mg} \mathrm{g}^{-1}$ de pó de casca de ovo desprovidas de membranas, como as analisadas neste estudo. Teores inferiores também são relatados na literatura (VILAR et al., 2010; BRUN et al., 2013), mas tratam-se de amostras contendo as membranas proteicas que se encontram aderidas à casca, ocasionando a redução da concentração mineral do material. Além disso, neste estudo, a concentração de Ca foi semelhante entre as cascas de ovo de granja e coloniais. Este 
Tabela 1 - Conteúdo de minerais em pós de cascas de ovo de granja e coloniais (média \pm erro padrão).

\begin{tabular}{|c|c|c|c|}
\hline \multicolumn{4}{|c|}{ 2 } \\
\hline & Brancos $(n=11)$ & Vermelhos $(n=9)$ & Cascas de ovo coloniais $(n=8)$ \\
\hline \multicolumn{4}{|c|}{ Minerais essenciais } \\
\hline $\mathrm{Ca}\left(\mathrm{mg} \mathrm{g}^{-1}\right)$ & $367,4 \pm 2,5$ & $368,2 \pm 1,5$ & $362,0 \pm 3,0$ \\
\hline $\operatorname{Mg}\left(\mathrm{mgg} \mathrm{g}^{-1}\right)$ & $3,08 \pm 0,04$ & $3,04 \pm 0,03$ & $3,65 \pm 0,13 *$ \\
\hline $\mathrm{Fe}\left(\mu \mathrm{gg}^{-1}\right)^{\#}$ & $<1,00-5,13$ & $<1,00$ & $<1,0-6,25$ \\
\hline $\mathrm{Cr}\left(\mu \mathrm{gg}^{-1}\right)$ & $<0,3$ & $<0,3$ & $<0,3$ \\
\hline $\operatorname{Mn}\left(\mu g^{-1}\right)$ & $<0,2$ & $<0,2$ & $<0,2$ \\
\hline $\operatorname{Mo}\left(\mu \mathrm{g} \mathrm{g}^{-1}\right)$ & $<0,2$ & $<0,2$ & $<0,2$ \\
\hline $\mathrm{Ni}\left(\mu \mathrm{g} \mathrm{g}^{-1}\right)$ & $<0,6$ & $<0,6$ & $<0,6$ \\
\hline $\operatorname{Se}\left(\mu g^{-1}\right)$ & $<0,8$ & $<0,8$ & $<0,8$ \\
\hline \multicolumn{4}{|c|}{ Minerais não-essenciais } \\
\hline $\operatorname{Sr}\left(\mathrm{mg} \mathrm{g}^{-1}\right)$ & $0,53 \pm 0,02$ & $0,60 \pm 0,04$ & $0,37 \pm 0,04 *$ \\
\hline $\mathrm{Al}\left(\mu \mathrm{g} \mathrm{g}^{-1}\right)$ & $<0,7$ & $<0,7$ & $<0,7$ \\
\hline $\mathrm{Cd}\left(\mu \mathrm{g} \mathrm{g}^{-1}\right)$ & $<0,02$ & $<0,02$ & $<0,02$ \\
\hline $\mathrm{Pb}\left(\mu \mathrm{g} \mathrm{g}^{-1}\right)$ & $<0,2$ & $<0,2$ & $<0,2$ \\
\hline
\end{tabular}

* Valores estatisticamente diferentes dos ovos de granja de acordo com o teste de Duncan $\mathrm{P}<0,05$ ).

\# Expresso em intervalo de concentração. Quantificado apenas em 3 amostras das cascas de ovo de granja branco e em 2 amostras das cascas de ovo coloniais.

resultado está em concordância com estudos prévios que demonstraram níveis constantes de Ca em cascas de ovo de poedeiras de sistemas de criação e dietas variadas (SCHAAFSMA et al., 2000; KÜÇÜKYILMAZ et al., 2012; BRUN et al., 2013). Sabe-se que os ovos coloniais normalmente apresentam cascas mais espessas do que as dos ovos de granja, podendo apresentar maior proporção de $\mathrm{Ca}$ na casca em relação ao peso total do ovo (HOLT et al., 2011). No entanto, como avaliamos a concentração do mineral por grama de casca e não em relação ao ovo em si, essa diferença não pode ser observada.

Os teores de $\mathrm{Mg}$ nas cascas variaram de 3,04 a 3,65 $\mathrm{mg} \mathrm{g}^{-1}$ (Tabela 1), em concordância com estudos prévios (SCHAAFSMA et al., 2000; VILAR et al., 2010; KÜÇÜKYILMAZ et al., 2012). Apesar do Mg ser o segundo mineral mais abundante nas cascas de ovo, a quantidade fornecida pelas cascas não é expressiva. Enquanto o consumo de $2,7 \mathrm{~g}$ de casca de ovo fornece $100 \%$ da ingestão diária recomendada (IDR) de $\mathrm{Ca}$ para adultos (IDR=1000mg; BRASIL, 2003b), essa quantidade do produto forneceria menos de $4 \%$ da IDR para o Mg (IDR=260mg; BRASIL, 2003b). Ainda assim, notou-se uma variação no teor deste mineral entre os tipos de cascas. As cascas de ovo coloniais apresentaram teores de $\mathrm{Mg}$ aproximadamente $20 \%$ superiores em comparação às cascas de ovo de granja, tanto brancas quanto vermelhas (Tabela 1; $\mathrm{P}<0,05)$. KÜÇÜKYILMAZ et al. (2012) observaram pequeno aumento de $\mathrm{Mg}(10 \%)$ em cascas de ovo de poedeiras de sistema de criação orgânico em comparação às mantidas confinadas, atribuindo esse resultado ao livre acesso dessas poedeiras ao solo e pequenas pedras contendo $\mathrm{Mg}$. Por outro lado, SCHAAFSMA et al. (2000) e BRUN et al. (2013) encontraram quantidades semelhantes de $\mathrm{Mg}$, independente do sistema de criação, possivelmente em função do material disponível no ambiente em que as poedeiras estavam sendo criadas.

Além do $\mathrm{Ca}$ e $\mathrm{Mg}$, foi detectado de 0,37 a $0,60 \mathrm{mg} \mathrm{g}^{-1}$ de Sr nas cascas de ovo, sendo que as amostras de granja apresentaram teores superiores em comparação às coloniais (Tabela 1; $\mathrm{P}<0,05$ ). Este mineral não é considerado essencial a humanos, mas é utilizado no tratamento da osteoporose, sendo associado ao aumento da formação, redução da reabsorção e melhora da microarquitetura óssea (UNFER et al., 2007; BOYD et al., 2011). A ingestão diária normal de $\mathrm{Sr}$ situa-se entre 1 a $3 \mathrm{mg}$ e, conforme SCHAASFSMA et al. (2000), o consumo do pó da casca de ovo como fonte de $\mathrm{Ca}$ acarretaria em aumento de 15 a $40 \%$ nesta ingestão. Embora a quantidade de $\mathrm{Sr}$ fornecida pela casca de ovo esteja muito abaixo das doses terapêuticas, ela poderia trazer efeitos benéficos à saúde óssea em longo prazo (SCHAASFSMA et al., 2002). Estudos prévios apontam teores de $\mathrm{Sr}$ variando de 0,1 até $1 \mathrm{mg} \mathrm{g}^{-1}$ no pó de casca de ovo (SCHAASFSMA et al., 2000; VILAR et al., 2010; BRUN et al., 2013), variação que, assim como a deste estudo, 
podem ser explicadas por diferenças na dieta das poedeiras, já que o teor de $\mathrm{Sr}$ depositado na casca de ovo é diretamente relacionado à sua ingestão (SCHAASFSMA et al., 2000). Sugere-se que a maior concentração de $\mathrm{Sr}$ encontrada nas cascas de granja em comparação às coloniais seja em decorrência das dietas destas poedeiras. Enquanto as poedeiras de granja possuem dieta controlada, frequentemente suplementada com fontes minerais que poderiam conter teores mais elevados de $\mathrm{Sr}$, as poedeiras coloniais são alimentadas somente com hortaliças e cereais.

Os microminerais $\mathrm{Cr}$, $\mathrm{Mn}, \mathrm{Mo}, \mathrm{Ni}$, Se e Al não foram detectados neste estudo (Tabela 1). Do mesmo modo, estudos prévios não detectaram esses minerais ou os detectaram em concentrações muito baixas (ng a poucos $\mu \mathrm{g} \mathrm{g}^{-1}$; SCHAASFSMA et al., 2000; VILAR et al., 2010). O micromineral Fe foi detectado apenas em cinco amostras, atingindo o teor máximo de $6,25 \mu \mathrm{g} \mathrm{g}^{-1}$ (Tabela 1). Estudos prévios relatam teores variados, de 1,5 até $23 \mu \mathrm{g} \mathrm{g}^{-1}$ de Fe na casca de ovo, denotando que a concentração deste mineral pode variar nas cascas (SCHAAFSMA et al., 2000; VILAR et al., 2010; KÜÇÜKYILMAZ et al., 2012).

A segurança de fontes naturais de cálcio para o consumo humano vem sendo questionada, já que elas podem conter quantidades relevantes de minerais tóxicos (KIM, 2004; MATTOS et al., 2006). No presente estudo, não foram detectados $\mathrm{Cd}$ ou $\mathrm{Pb}$ em amostras de cascas de ovo provenientes da região central do Rio Grande do Sul. De forma semelhante, VILAR et al. (2010) e SANTOS et al. (2012) não detectaram contaminação por $\mathrm{Cd}$, $\mathrm{Pb}$ ou $\mathrm{Hg}$ em amostras de cascas de ovos oriundas de Sorocaba-SP e Aracaju-Se, respectivamente.
Segundo SCHAAFSMA et al. (2000), a casca de ovo pode ser vantajosa em relação a outras fontes de $\mathrm{Ca}$, já que não foram detectados $\mathrm{Cd}$ ou $\mathrm{Hg}$ em amostras provenientes da Eslováquia, enquanto o $\mathrm{Ca}$ de ostras e o $\mathrm{CaCO}_{3}$ purificado apresentaram contaminação por estes metais.

As amostras de pó de cascas de ovo analisadas apresentaram condições sanitárias satisfatórias, quando comparadas ao padrão microbiológico estabelecido para ovos (BRASIL, 2001; Tabela 2). A lavagem inicial, seguida de fervura em água por 10min (tratamento 2) foi tão eficiente quanto o tratamento incluindo a imersão em hicloclorito a $1 \%$ para higienização das cascas de ovo (tratamento 1). De acordo com NAVES et al. (2007), o procedimento de higienização proposto também é eficiente para obter condições sanitárias satisfatórias para mesófilos aeróbios, bolores e leveduras.

\section{CONCLUSÃO}

A casca de ovo pode ser considerada abundante em $\mathrm{Ca}$, já que apenas $2,7 \mathrm{~g}$ do pó fornecem a quantidade diária recomendada desse mineral para um adulto. As amostras coloniais e de granja, brancas e vermelhas, apresentaram teores similares de $\mathrm{Ca}$, mas as cascas de ovo de granja apresentaram maior concentração de $\mathrm{Mg}$ e menor concentração de $\mathrm{Sr}$ que as coloniais. Não foram encontradas quantidades significativas de outros minerais essenciais ou tóxicos nas cascas de ovo. Além disso, a contaminação microbiana não é um fator de restrição ao uso da casca de ovo por humanos, já que é possível obter pó de casca de ovo seguro em relação ao caráter higiênico-sanitário utilizando fervura em água e secagem a $50^{\circ} \mathrm{C}$ por $24 \mathrm{~h}$.

Tabela 2 - Análises microbiológicas de cascas de ovo submetidas a diferentes processos de higienização.

\begin{tabular}{|c|c|c|c|}
\hline \multirow{2}{*}{ Patógeno } & \multicolumn{3}{|c|}{---Método de higienização-- } \\
\hline & Tratamento $1(\mathrm{n}=3)$ & Tratamento $2(\mathrm{n}=3)$ & Padrão microbiológico \\
\hline Coliformes totais $\left(\mathrm{NMP} \mathrm{g}^{-1}\right)$ & $<3,0$ & $<3,0$ & $-*$ \\
\hline Coliformes termotolerantes (NMP g ${ }^{-1}$ ) & $<3,0$ & $<3,0$ & 10 (máx.) \\
\hline Staphylococcus coagulase positiva (UFC $\left.\mathrm{g}^{-1}\right)$ & $<1 \times 10^{1}$ & $<1 \times 10^{1}$ & $5 \times 10^{2}$ (máx.) \\
\hline Salmonella sp. (em 25g de amostra) & Ausência & Ausência & Ausência \\
\hline
\end{tabular}

Tratamento 1: $5 \mathrm{~min}$. de imersão em hipoclorito de sódio 1\% e 10min. de fervura com água.

Tratamento 2: 10min. de fervura com água.

$\mathrm{NMP} \mathrm{g}^{-1}$ : número mais provável por grama de amostra.

$\mathrm{NMP} \mathrm{g}^{-1}$ : unidades formadoras de colônia por grama de amostra.

* padrão microbiológico não estabelecido para ovos. 


\section{AGRADECIMENTOS}

Os autores agradecem ao programa Fundo de Incentivo à Pesquisa (FIPE) da Universidade Federal de Santa Maria (UFSM), pelos recursos concedidos, e à Coordenação de Aperfeiçoamento de Pessoal de Nível Superior (CAPES), pela concessão de bolsa de Mestrado.

\section{REFERÊNCIAS}

AWUMEY, E.M.; BUKOSKI R.D. Cellular functions and fluxes of calcium. In: WEAVER C.M.; HEANEY, R.P. Calcium in human health. Totowa: Springer, 2006. Cap.3, p.13-35.

BOYD, S.K. et al. Increased bone strength is associated with improved bone microarchitecture in intact female rats treated with strontium ranelate: a finite element analysis study. Bone, v.48, n.5, p.1109-1116, 2011. Disponível em: <http://www.ncbi. nlm.nih.gov/pubmed/21276882>. Acesso em: 19 mar. 2014. doi: 10.1016/j.bone.2011.01.004.

BRASIL. Ministério da Agricultura do Abastecimento e da Reforma Agrária. Secretaria de Defesa Agropecuária. Instrução normativa $\mathrm{n}^{\mathrm{o}}$ 62, de 26 de agosto de 2003a. Métodos analíticos oficiais para análises microbiológicas para controle de produtos de origem animal e água. Diário Oficial da República Federativa do Brasil, Brasília, DF, 18 set. 2003a. Seção 1, p.14.

BRASIL. Ministério da Saúde. Agência Nacional de Vigilância Sanitária. Resolução no 360, de 23 de dezembro de 2003b. Regulamento técnico sobre rotulagem nutricional de alimentos embalados. Disponível em: <http://portal.anvisa. gov.br/wps/wcm/connect/ec3966804ac02cf1962abfa337abae9d/ Resolucao_RDC_n_360de_23_de_dezembro_de_2003. pdf?MOD=AJPERES $>$. Acesso em: 30 abr. 2014.

BRASIL. Ministério da Saúde. Agência Nacional de Vigilância Sanitária. Resolução RDC nº 12, de 2 de janeiro de 2001. Regulamento técnico sobre padrões microbiológicos para alimentos. Diário Oficial da República Federativa do Brasil, Brasília, DF, 10 jan. 2001. Seção 1, p.45.

BRUN, L.R. et al. Chicken eggshell as suitable calcium source at home. International Journal of Food Sciences and Nutrition, v.64, n.6, p.740-743, 2013. Disponível em: <http://www.ncbi.nlm. nih.gov/pubmed/23607686>. Acesso em: 23 set. 2014. doi:10.310 9/09637486.2013.787399.

FISBERG, R.M. et al. Ingestão inadequada de nutrientes na população de idosos do Brasil: Inquérito Nacional de Alimentação 2008-2009. Revista de Saúde Pública, v.47, p.S222-S230, 2013. Disponível em: <http://www. scielo.br/scielo.php? script $=$ sci_arttext\&pid=S0034$89102013000700008 \& \operatorname{lng}=\mathrm{em}>$. Acesso em: 2 jan. 2014. doi: 10.1590/S0034-89102013000700008.

HOLT, P.S. et al. The impact of different housing systems on egg safety and quality. Poultry Science, v.90, n.1, p.251262, 2011. Disponível em: <http://www.ncbi.nlm.nih.gov/ pubmed/21177467>. Acesso em: 22 mar. 2014. doi:10.3382/ ps.2010-00794.

KIM, M. Mercury, cadmium and arsenic contents of calcium dietary supplements. Food Additives and
Contaminants, v.21, n.8, p.763-767, 2004. Disponível em: <http://www.ncbi.nlm.nih.gov/pubmed/15370826>. Acesso em: 9 jun. 2014. doi: 10.1080/02652030410001713861.

KING'ORI, A.M. A review of the uses of poultry eggshells and shell membranes. International Journal of Poultry Science, v.10, n.11, p.908-912, 2011. Disponível em: <http://www.pjbs.org/ ijps/fin2058.pdf>. Acesso em: 19 mar. 2014.

KÜÇÜKYILMAZ, K. et al. Effect of an organic and conventional rearing system on the mineral content of hen eggs. Food Chemistry, v.132, n.2, p.989-992, 2012. Disponível em: <http:// www.sciencedirect.com/science/article/pii/S0308814611016669>. Acesso em: 19 mar. 2014. doi:10.1016/j.foodchem.2011.11.084.

MAEHIRA, F. Effects of calcium sources and soluble silicate on bone metabolism and the related gene expression in mice. Nutrition, v.25, n.5, p.581-589, 2009. Disponível em: <http:// www.sciencedirect.com/science/article/pii/S0899900708004541>. Acesso em: 9 jun. 2014. doi:10.1016/j.nut.2008.10.023.

MATTOS, J.C.P. et al. Lead content of dietary calcium supplements available in Brazil. Food Additives \& Contaminants, v.23, n.2, p.133-139, 2006. Disponível em: <http://www.ncbi. nlm.nih.gov/pubmed/16449055>. Acesso em: 06 abr. 2014. doi:10.1080/02652030500316959.

MINE, Y. Egg bioscience and biotechnology. New Jersey: Wiley, 2008. 362p.

NAVES, M.M.V. Pó da casca de ovo como fonte de cálcio: qualidade nutricional e contribuição para o aporte adequado de cálcio. Publicação semestral da Pró-Reitoria de Extensão e Cultura da Universidade Federal de Goiás, v.5, n.2, p.24-26, 2003. Disponível em: <http://www.proec.ufg.br/revista_ufg/fome/ casca.html $>$. Acesso em: 23 set. 2014.

NAVES, M.M.V. et al. Avaliação microbiológica do pó de casca de ovo e otimização da técnica de elaboração do produto. Pesquisa Agropecuária Tropical, v.37, n.2, p.113-118, 2007. Disponível em: <http://www.revistas.ufg.br/index.php/pat/article/ view/1836/1748>. Acesso em: 19 mar. 2014.

OLIVEIRA, D.A. et al. A literature review on adding value to solid residues: egg shells. Journal of Cleaner Production, v.46, p.42-47, 2013. Disponível em: <http://linkinghub.elsevier.com/ retrieve/pii/S0959652612005161>. Acesso em: 19 mar. 2014. doi:10.1016/j.jclepro.2012.09.045.

ROVENSKÝ, J. et al. Eggshell calcium in the prevention and treatment of osteoporosis. International Journal of Clinical Pharmacology Research, v.23, n.2, p.83-92, 2003.

SANTOS, S.T.S. et al. Análises dos constituintes inorgânicos da casca do ovo. Scientia Plena, v.8, n.3, p.1-4, 2012. Disponível em: <http://www.scientiaplena.org.br/ojs/index.php/sp/article/ viewFile/917/476>. Acesso em: 27 mar. 2014.

SCHAAFSMA, A. et al. Positive effects of a chicken eggshell powder-enriched vitamin-mineral supplement on femoral neck bone mineral density in healthy late post-menopausal Dutch women. British Journal of Nutrition, v.87, n.3, p.267-275, 2002. Disponível em: <http://www.journals.cambridge.org/abstract 
S0007114502000570>. Acesso em: 25 fev. 2014. doi: 10.1079/ BJN2001515.

SCHAAFSMA, A. et al. Mineral, amino acid, and hormonal composition of chicken eggshell powder and the evaluation of its use in human nutrition. Poultry Science, v.79, n.12, p.18331838, 2000. Disponível em: <http://ps.oxfordjournals.org/ cgi/doi/10.1093/ps/79.12.1833>. Acesso em: $12 \mathrm{dez} .2013$. doi:10.1093/ps/79.12.1833.

SCHULZE, K.J. Calcium. In: CABALLERO B. et al. Encyclopedia of human nutrition. 3.ed. Amsterdam: Elsevier, 2013. p.228-234.
SILVA, A.G.H.; COZZOLINO, S.M.F. Cálcio. In: COZZOLINO, S.M.F. Biodisponibilidade de nutrientes. 2.ed. Barueri: Manole, 2007. Cap.22, p.456-481.

UNFER, T.C. et al. $\mathrm{Sr}$ and Fe relationship with hormone replacement therapy and bone mineral density. Clinica Chimica Acta, v.384, n.1-2, p.113-117, 2007. Disponível em: <http://www. sciencedirect.com/science/article/pii/S000989810700335X>. Acesso em: 6 abr. 2014. doi: 10.1016/j.cca.2007.06.010.

VILAR, J.S. et al. Composição química da casca de ovo de galinha em pó. Boletim do Centro de Pesquisa de Processamento de Alimentos, v.28, n.2, p.247-254, 2010. Disponível em: $<$ http://ojs. c3sl.ufpr.br/ojs2/index.php/alimentos/article/view/20439/13698>. Acesso em: 24 mar. 2014.

Ciência Rural, v.45, n.3, mar, 2015. 